www.jmscr.igmpublication.org Impact Factor 5.244

Index Copernicus Value: 83.27 ISSN (e)-2347-176x ISSN (p) 2455-0450 crossref DOI:_http://dx.doi.org/10.18535/jmscr/v4i7.08

\title{
Efficacy of Proprioceptive Neuromuscular Facilitation techniques to Pelvic girdle muscles in improving gait in hemiplegic patients
}

\author{
A.Thiruppathi M.P.T. ${ }^{1}$, P.Rajitha M.P.T ${ }^{2}$, V.Kiran M.P.T. ${ }^{3}$, K.Suneel kumar M.P.T. ${ }^{4}$ \\ ${ }^{1}$ Professor, Narayana College of Physiotherapy, ${ }^{2}$ Clinical Therapist, Department of Physiotherapy \\ ${ }^{3}$ Professor, Narayana College of Physiotherapy, ${ }^{4}$ Professor, Narayana College of Physiotherapy \\ Narayana College of Physiotherapy, Nellore
}

\begin{abstract}
Objectives: To compare the effects of PNF treatment applied to the pelvic region in patients with hemiplegia acute and chronic stage and investigate whether the duration of hemiplegia affected the results of the treatment.

Study Design: Quasi-experimental design.

Source of Data: Subjects are chosen from the Department of Neurology, Narayana Medical College \& Hospital, Nellore.

Results: In this study, both subjects with acute and chronic hemiplegia had a mean age of $56.8 \pm 8.0$ years. Chronic hemiplegic patients have had hemiplegia since $15.4 \pm 1.7$ months. All subjects were at brunnstorm stage 3, 4 or 5. There was no difference between both groups in case of age, gender, side of hemiplegia and brunnstorm stage. In the group with acute hemiplegia, each subject demonstrated increasing gait speed and cadence after treatment. Also in the group with chronic hemiplegia, each subject demonstrated an increase in gait speed and cadence after treatment more or less similar to acute patients. For Patients in both groups, both mean speed and cadence after treatment greater than pretest values.

Conclusion: Both acute and chronic hemiplegic patient groups have shown improvement from Pre-test to Post-test equally. This observation lends support to the null hypothesis that the duration of hemiplegia may not be as crucial for functional recovery as previously thought, at least relative to the gait indexes of recovery was examined.

Keywords: Acute \& Chronic Hemiplegia, PNF Techniques, pelvic floor muscles, Gait pattern
\end{abstract}

\section{INTRODUCTION}

Stroke is a clinical disorder, reporting a range of issue which brings in focal cerebral ischemia ${ }^{1}$. Stroke is the significant reason for the passing in industrialized nations $10-12 \%$ is because of stroke, and around $88 \%$ of the passings ascribed to stroke are amongst individuals more than 65 years of age ${ }^{2}$. Stroke is accounted for a greater significance of illness in the community. In persons with hemiplegic, posture, tone and coordinated reciprocal movements, which are required for normal gait but they are usually impaired.

Normal reciprocal pelvic movement is frequently restored by a fixed pelvic retraction which makes it troublesome for patients to swing the influenced lower limb forward ${ }^{3}$. The resultant walking pattern is moderate, with a short stride length and 
unbalanced strides. Frequently called hemiplegic walk, this moderate step can be seen in clinical settings as a decline in walking pace and rhythm. Change of the nature of step is frequently a primary aim of exercise based rehabilitation for patients with hemiplegia. Proprioceptive neuromuscular facilitation (PNF) is one approach generally used to enhance the gait pattern of patients with hemiplegia ${ }^{4,5}$. Different PNF procedures have been utilized, contingent upon the influenced site. Among these PNF strategies is the help of pelvic motion to enhance control of the pelvis. Since the pelvis has been depicted as a "key purpose of control" for keeping up a walking design, techniques intended to influence the pelvis are broadly used ${ }^{6}$. A small number of studies, however, have reported the therapeutic outcome of facilitation of pelvic motion in patients with hemiplegia.

Apart from the type and duration of PNF treatments, the duration of the hemiplegia should be considered a factor affecting functional recovery ${ }^{7,8,9}$. Although some clinicians contend that PNF treatments have little benefit for patients with hemiplegia of more than 6 months duration, others argue that the treatment should be administered as long as it contributes to functional improvement, regardless of the duration of hemiplegia. The evidence required to settle this disagreement is still lacking.

There is a need to develop an effective treatment pattern to improve gait and dynamic balance in hemiplegic patients with acute and chronic. This study is mainly intended to compare the effects of PNF treatments applied to the pelvic region in patients with hemiplegia acute and chronic stage. The research hypothesis was there was an effect of a PNF technique to pelvic girdle muscles in improving gait in patients with acute and chronic hemiplegia. Duration of hemiplegia affects the results of the treatments.

\section{METHODOLOGY}

All patients authenticate an informed consent form, endorsed by the Narayana Medical
Institutional ethical committee. This study includes thirty patients who were the age of 45 to 60 years admitted in department Neurology $\mathrm{NMCH}$. This research work was conducted as a randomized sampling with a (2x2) (Group $\mathrm{x}$ measure) measures Quasi-experimental design. To be eligible for this study, patients had to have a diagnosis of hemiplegia secondary to a cerebrovascular accident, with symptoms having lasted either less than a month or more than 6 months. The capacity to walk no less than $3 \mathrm{~m}$ (60 $\mathrm{ft}$ ) without manual help, with or without an assistive gadget, at one's particular velocity. The patient had intact kinesthesia for the hip, knee, and ankle and who had no proprioceptive deficits or visual problems. Patients should have a lower limb motor recovery of brunnstom 3,4 or 5 stages 10,11. This study excluded Hemodynamic instability, severe deformities, recent surgeries, Noncooperative and mentally unstable persons and Vestibular disorders. All our patients were equally and randomly divided into Acute Hemiplegic Group (AHG) and chronic Hemiplegic Group (CHG). Basic demographic data obtained through interview and medical chart review.

\section{PROCEDURE}

In this study 30 patients were selected according to the inclusion criteria, these patients were randomly assigned into groups Acute Hemiplegic Group (AHG) and chronic Hemiplegic Group (CHG) and 15 patients in each group. All patients were assessed before pre-test (1st week) and after completion of 4 weeks post-test with timed up and go test and cadence. The treatment in this study consists of facilitation of pelvis in anterior elevation and posterior depression in D1, D2 lower extremity patterns. The following techniques were in 10 minutes each, rhythmic initiation in D1 LE pattern, slow reversal in D1 LE pattern, agonistic reversal in D1 LE pattern, rhythmic initiation in D2 LE pattern, slow reversal in D2 LE pattern and agonistic reversal in D2 LE pattern ${ }^{12}$. These procedures were done to 
facilitate anterior elevation and posterior depression of pelvic movement in side-lying position ${ }^{13}$. Each treatment session lasted 30 minutes, with a total of 16 treatment sessions four times a week for 4 weeks. The elements of PNF, such as manual contact, stretch, resistance, and verbal cueing, were incorporated into the treatment scheme ${ }^{14,15}$. The validity of the PNF technique and the reliability of treatment procedures was established by setting an agreement score of greater than 95\% from their checklists. No other treatments of physical therapy were given to these patients throughout the period of this study.

Each test session included Timed up and go test, The total time in seconds and a total number of steps during the trial were obtained with a stopwatch and by counting heel-strikes, respectively. Gait speed was calculated as the distance three meters divided by the time $(\mathrm{m} / \mathrm{Sec})$ and cadence were calculated as the number of steps divided by the time (steps/min). For each patient, data obtained immediately prior to the treatment served as the control values, and data obtained after 16th treatment session served as experimental values. For each patient, data obtained immediately prior to the treatment served as the control values, and data obtained after $16^{\text {th }}$ treatment session served as experimental values. These scores were taken for further analysis. Data collected were analysed using Graph pad Prism-4 \& SPSS version 11.

\section{RESULTS}

In this study, both group patients with AHG and CHG had a mean age of $56.8 \pm 8.0$ years. Chronic hemiplegic $(\mathrm{CHG})$ patients have had hemiplegia since $15.4 \pm 1.7$ months. All patients were at brunnstorm stage 3,4 or 5 . There was no difference between both groups in case of age, gender, side of the hemiplegia and brainstorm stage of 3,4,5. In AHG group each subject demonstrated increasing gait speed and cadence after treatment. The group with $\mathrm{CHG}$, each member demonstrated an increase in gait speed and cadence after treatment more or less similar to AHG group. For patients in both groups, both mean speed and cadence after treatment greater than pre-test values.

TABLE:1 TUG-test for difference of means of gait speed

\begin{tabular}{|l|l|l|l|l|l|l|}
\hline \multirow{2}{*}{ Group } & \multicolumn{2}{|l|}{ Mean } & Variance & $\begin{array}{l}\text { T- Calculated } \\
\text { Value }\end{array}$ & $\begin{array}{l}\text { T- tabulated } \\
\text { Value }\end{array}$ & P(T<=t) \\
\cline { 2 - 5 } & Pre & Post & & 0.140 & 2.048 & 0.889 \\
\hline Post AHG- Group & 0.462 & 0.546 & 0.0097 & 0.0118 & & \\
\hline Post CHG- Group & 0.473 & 0.552 & 0.018 (AHG\& CHG) \\
*Post test values of gait speed were significant difference from pre test in both groups in (AHG) \\
P< $\mathbf{0 . 0 0 1}$
\end{tabular}

Figure 1:

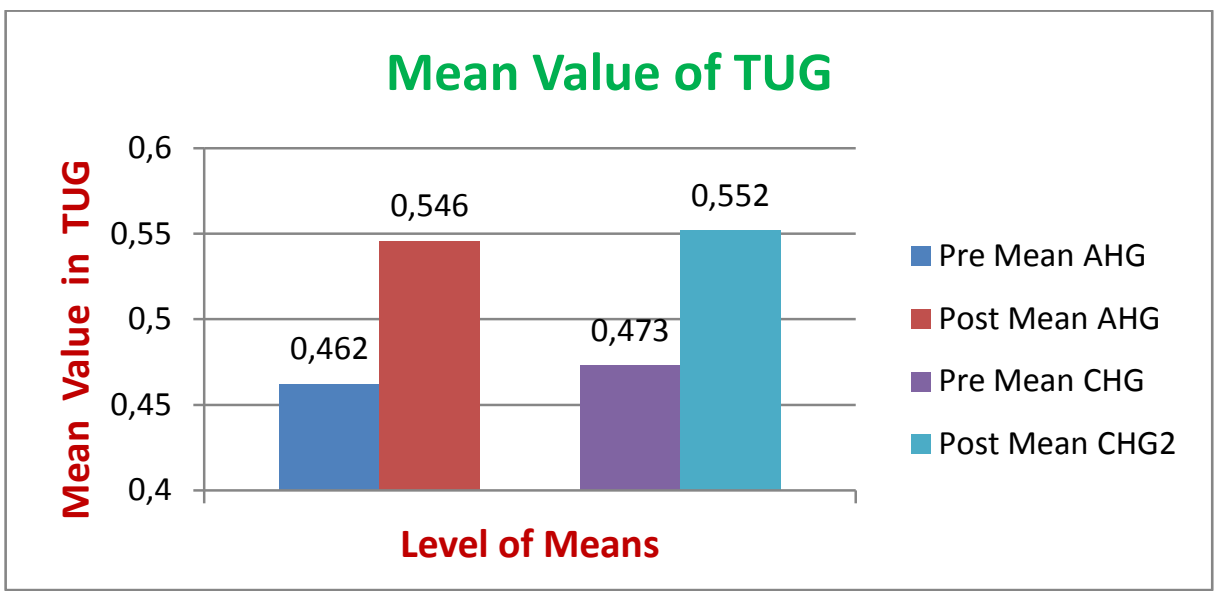


TABLE:2 Cadence speed for difference of means

\begin{tabular}{|l|l|l|l|l|l|l|}
\hline \multirow{2}{*}{ Group } & \multicolumn{2}{|l|}{ Mean } & Variance & $\begin{array}{l}\text { T- Calculated } \\
\text { Value }\end{array}$ & $\begin{array}{l}\text { T- tabulated } \\
\text { Value }\end{array}$ & P(T<=t) \\
\cline { 2 - 5 } & Pre & Post & & & 2.048 & 0.889 \\
\hline Post AHG- Group & 62.7 & 69.8 & 0.0097 & 0.140 & 2.04 & \\
\hline Post CHG- Group & 61.3 & 68.6 & 0.0118 & & \\
*Post test values of cadence were significant difference from pre test in both groups in \\
(AHG\& CHG) P< 0.001
\end{tabular}

Figure 2:

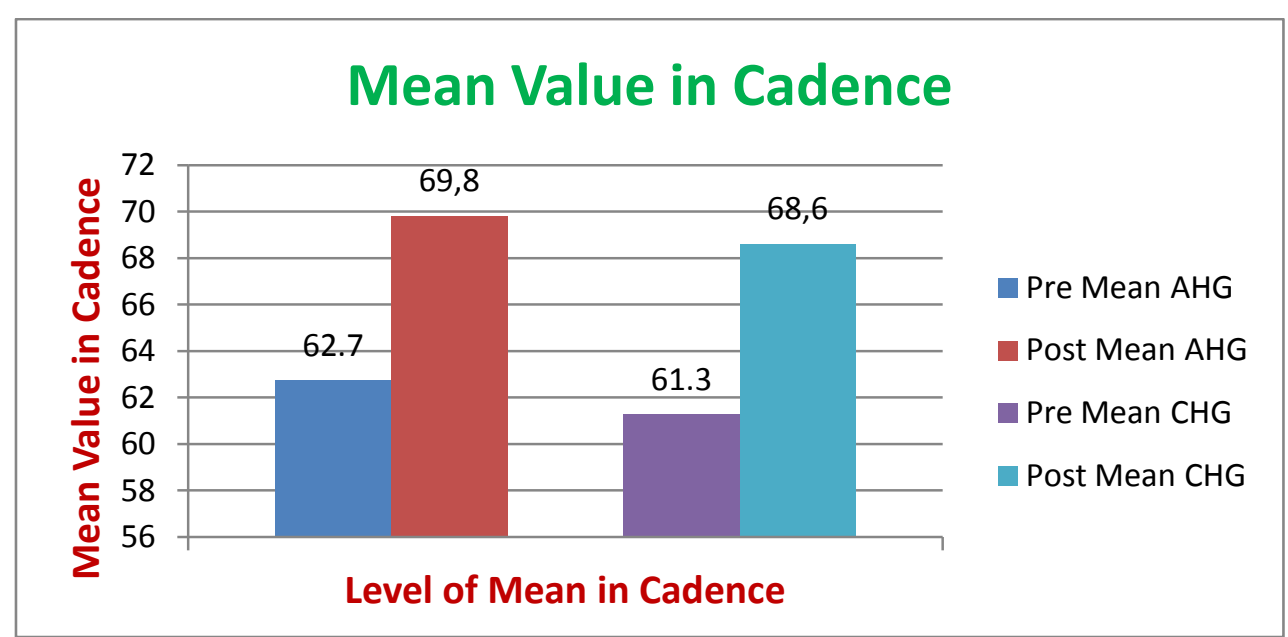

Since p-value (0.658) is more than the level of significance, We reject the alternative hypothesis or accept the null hypothesis.i.e, both groups have shown the same results.

\section{DISCUSSION}

The results of this study indicate that patients with hemiplegic benefitted from specific PNF pelvic techniques. Patients with acute hemiplegia showed immediate improvements both in gait speed and cadence ${ }^{16}$. Studies on the effectiveness of PNF on pelvic girdle have been both conflicting and supportive. Marek S M et al ${ }^{17}$ found that there is no immediate effect of resisting PNF pelvic exercise. This study both gait speed and cadence increased may be due to differences in PNF technique and duration of treatment. In this study Rhythmic Initiation, Slow Reversals, Agonistic Reversals applied for $30 \mathrm{~min}$ to facilitate pelvic movements. Immediate improvements in gait in chronic hemiplegic patients not found.

The difference in the effect of treatment between acute and chronic hemiplegic patients may be due to neural and structural changes, subjects with chronic hemiplegia most likely had been using abnormal gait patterns ${ }^{18}$. Changes in muscles that were excessively used in these gait patterns could influence the outcome of PNF treatment. Neural and structural changes in the muscles of patients with hemiplegia of long duration may account for the lack of an immediate effect from pelvic PNF treatment ${ }^{19}$.

After 16 PNF sessions, both gait speed and cadence improved in the patients with hemiplegia of long duration. The cumulative treatment effects were also clear in the results from the patients with hemiplegia of short duration after sixteen PNF sessions. One interesting finding of my study is that there was no difference in cumulative treatment effects between the two groups of subjects.

This observation lends support to the hypothesis that the duration of hemiplegia may not be as crucial for functional recovery as previously thought, at least relative to the gait indexes of recovery we examined. The improvement in gait speed after 16 PNF sessions was due to an increase in cadence accompanied by an increase in average stride length in $70 \%$ and $60 \%$ of the patients with hemiplegia of acute and chronic 
duration. Increases in cadence accounted for patients in both groups.

Patients with hemiplegia usually have slower gait speeds and shorter stride lengths than do subjects without hemiplegia. The limitation in gait speed and stride length is associated with the efficiency of advancing paretic limb in swing and in shifting weight to the paretic limb. Whether the improvements recorded in this study were due to a more efficient limb swing or due to a better single-limb balance is not known. The detailed gait analysis would have been required to understand the basis for the improvements we observed.

\section{CONCLUSION}

Proprioceptive neuromuscular facilitation (PNF) is one approach commonly used to improve the gait of patients with hemiplegia ${ }^{20}$. Both acute and chronic hemiplegic patient groups have shown improvement from Pre-test to Post-test equally. This observation lends support to the null hypothesis that the duration of hemiplegia may not be as crucial for functional recovery as previously thought, at least relative to the gait indexes of recovery I examined. This study found a certain limitation, absence of a true control group who receive no PNF patterns. The total study population was very small in size. There was no follow- up results were included to show the effect of training. All patients are at brunnstrom stages of 3,4 , or 5 that means patients are already mobile. If we are increasing intervention weeks, Including true control group who receives no PNF patterns and complete gait analysis may give a clear indication of improvement.

Acknowledgment: We would like to thank the Head of the Department Neurology and Neurorehabilitation Physiotherapsy HOD A.Kiran M.P.T., and the entire rehabilitation team professionals in the units of Neurology, who please agree to assist with participant recruitment.

\section{BIBLIOGRAPHY}

1. Bobath B. Adult Hemiplegia: Evaluation and Treatment. London, England: William Heinemann Ltd; 1990:16144.

2. Klein DA, Stone WJ, ea. PNF training and physical function in assisted living older adults. Journal of aging and physical activity 2002 (10) 476-488

3. Ernst E: A review of stroke rehabilitation and physiotherapy.Stroke 1990 (21) 10811085

4. Stem PH, McDowell F, Miller JM, Robinson M. Effects of facilitation exercise techniques in stroke rehabilitation. Arch Phys Med Rehabil. 1970;51:526-531.

5. Quin CE. Observations on effects of PNF techniques in the treatment of hemiplegia. Rheum Phys Med 1971;11:186192.

6. Trueblood PR, Walker JM, Perry J, Gronley JK. Pelvic exercise and gait in hemiplegia. Phys Ther. 1989;69:3240.

7. Knutsson E, Richards C. Different types of distributed motor control in gait of hemiplegic patients. Brain 1979;102:403415

8. Wall JC, Ashburn A Assessment of gait disability in hemiplegics. Scand J Rehabil Med.1979;11:95-99.

9. Duncan PW, Badke MB. Stroke Rehabilitation: The Recovery of Motor Control NewYork, NY; Year Book Medical Publishers; 1987:85-121.

10. Brunnstrorn S. Movement Therapy in Hemiplegia.Hagerstown, Harper \& Row; 1992:34-100.

11. Nitz J, Burke B. A study of the facilitation. Perry J.The mechanics of walking in hemiplegia.Clin OTthop, 1969632331.

12. Buck M, Beckers D, Adler S: PNF in Practice. Springer Verlag/publishers 2008.

13. Dean CM, Richards CL, Malouin F. Taskrelated circuit training improves performance of locomotor tasks in chronic 
stroke: a randomized, controlled pilot trial. Arch Phys Med Rehabil. 2000; 81:

14. Kofotolis N, Eleftherios K: Effects of two 4-week PNF programs on muscle endurance,flexibility, and functional performance in women with CLBP. Physical Therapy 2006 (7) 1001-1012

15. Johnson GS, Johnson VS. The application of the principles and procedures of PNF for the care of lumbar spinal instabilities. The Journal of manual and Manipulative therapy 2002 (2) 83105

16. Wang RY. The immediate and cumulative effects of proprioceptive neuromuscular facilitation in case of patients with hemiplegia of long and short duration. Physical Therapy 1994 (12) 25-32

17. Marek SM ea. : Acute effects of static and PNF stretching on muscle strength and power output. Journal of athletic training 2005 (2) 94-103

18. Klein DA, Stone WJ, ea. PNF training and physical function in assisted living older adults. Journal of aging and physical activity 2002

19. Sharman MJ, Cresswell AG, Riek S: Prorioceptive Neuromuscular Facilitation Stretching, mechanisms and clinical implications. Sports Med 2006 (11) 929939

20. Knott M,Voss DE: Proprioceptive neuromuscular facilitation - patterns and techniques $2^{\text {nd }}$ edition. New York Haper \& Row publishers 1968 